\title{
Percutaneous Ethanol Injection Therapy for Recurrent Benign Thyroid Nodule : Report of a Case
}

\author{
Terumasa Kurihara $^{1,2}$ and Tsunehiro Ishida ${ }^{2}$
}

\begin{abstract}
We report a successfully treated case of recurrent benign thyroid nodule using percutaneous ethanol injection therapy (PEIT). The patient was a 64-year-old man who had undergone right lobectomy of the thyroid due to follicular adenoma 5 years previously. After surgery, a large mass of the anterior neck was discovered. Ultrasound revealed a large cystic nodule, $5.5 \mathrm{~cm}$ in diameter, in the left lobe of the thyroid. Although aspiration of cystic fluid was repeated five times, it returned rapidly to its initial level. Subsequently, compression symptoms such as swallowing disturbance and visible disfigurement occurred. After one trial of PEIT, tumor volume markedly decreased to $0.6 \mathrm{~cm}$ in diameter and compression symptoms disappeared. Therefore PEIT would appear to be a safe, simple, and effective procedure for recurrent benign thyroid nodules and may improve the patient's quality of life. (Kitakanto Med J 2004; $54: 223 \sim 225)$
\end{abstract}

Key words : percutaneous ethanol injection therapy, PEIT, benign thyroid tumor, thyroid cystic nodule, recurrence

\section{Introduction}

The recurrence of benign thyroid nodules is not a rare event, especially in cases of adenomatous goiter, which has the potential to occur multifocally in the thyroid gland. Commonly, reoperations for benign thyroid tumors after thyroidectomy are not performed due to the possibility of disfigurement and such complications as recurrent laryngeal nerve palsy and permanent tetany.

Percutaneous ethanol injection therapy (PEIT) is now widely available for the treatment of hepatocellular carcinoma and metastatic carcinoma of the liver. ${ }^{1}$ In the case of thyroid diseases, PEIT is used not only for benign tumors such as cystic nodules and autonomously functioning thyroid nodules (AFTN),${ }^{2-5}$ but also to treat inoperable thyroid cancer or local recurrence of cancer. ${ }^{6,7}$ We herein present a successfully treated case of recurrent benign thyroid nodule after thyroidectomy using one round of PEIT.

\section{Case report}

A 64-year-old man was admitted to our hospital because of a large neck mass. The patient had undergone right lobectomy of the thyroid 5 years previously and pathology confirmed follicular adenoma. After surgery, suppressive therapy of levothyroxine sodium (Thyradin-S ${ }^{\circledR} 50 \mu \mathrm{g} /$ day) was administered for 4 years. Five years after surgery, a mass in the left side of the neck was discovered. Ultrasound (US) revealed a predominantly cystic nodule, $5.5 \times 5.0 \mathrm{~cm}$ in size, in the remnant left lobe of the thyroid (Fig. 1A). Cytologic examination of aspirates from the cystic nodule showed no atypical cells. Fluid removal by aspiration was repeated five times at 4 to 6 week intervals; however fluid returned each time to previous levels. With the enlargement of the tumor, compression symptoms such as swallowing disturbances and neck discomfort reappeared and increased in severity. Since malignancy was ruled out from cytologic examinations and US showed the mass was predominantly cystic, we decided to perform PEIT under informed consent.

1 Department of Surgery, Keiaido Hospital, 504-6 Omama, Omama-machi, Gunma 376-0101, Japan

2 Department of Surgery, National Takasaki Hospital, 36 Takamatsu-cho, Takasaki, Gunma 370-0829, Japan

Received: May 13, 2004

Address: TERUMASA KURIHARA 504-6 Omama, Omama-machi, Gunma 376-0101, Japan Department of Surgery, Keiaido Hospital 


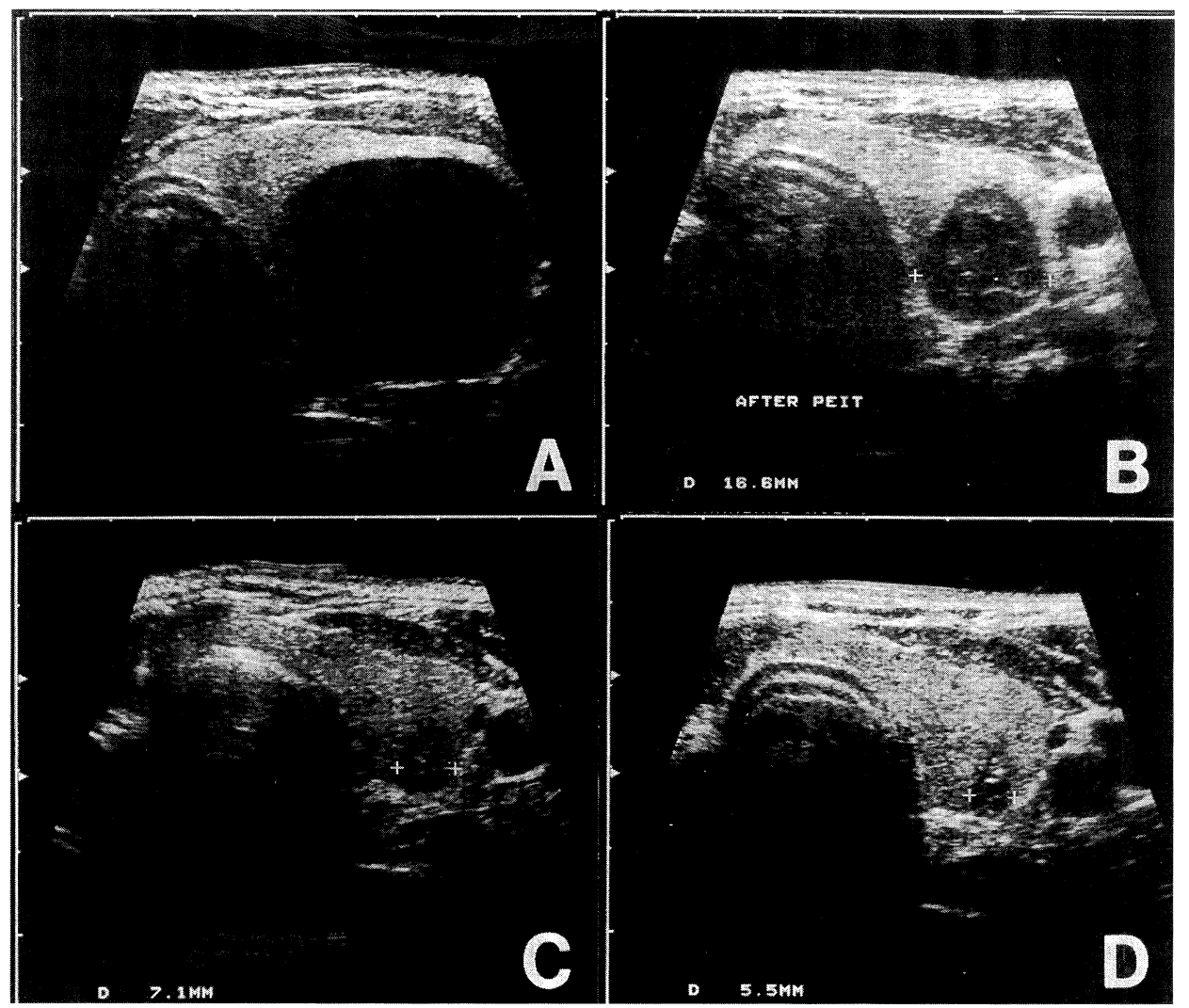

Fig. 1 Ultrasonograms of the thyroid before and after PEIT.

a : before PEIT

b : 1 month after PEIT

c : 10 months after PEIT

d : 16 months after PEIT

The patient was in a euthyroid state based on measurements of serum free triiodothyronine, free thyroxine, and thyroid-stimulating hormone.

\section{PEIT procedure}

We performed US examination by B-mode and color Doppler with a $7.5 \mathrm{MHz}$ probe. The tumor's size, shape, position, and depth from the skin and its relation to surrounding arteries and veins was carefully noted. After cleaning the skin with antiseptic and local anesthesia, an 18-gauge needle was inserted into the center of the nodule under US guidance. After complete fluid removal, $2 \mathrm{ml}$ of sterile absolute ethanol was then instilled without removing the needle. After confirmation of tissue coagulation as indicated by a hyperechoic change on US, the remaining fluid including injected ethanol was aspirated to the extent possible. Then the needle was carefully pulled out.

\section{Clinical course after PEIT}

Within one month of the PEIT, the palpable neck mass became very small $(1.6 \times 1.2 \mathrm{~cm})$ and predominantly solid in composition as indicated by US (Fig. 1B). Compression symptoms had disappeared. Ten months after PEIT the neck mass was impalpable, with US indicating it had decreased to $0.7 \times 0.7 \mathrm{~cm}$ in size with its cystic portion gone (Fig. 1C). At present, 16 months after the PEIT, US shows a very small solid mass, $0.6 \times 0.6 \mathrm{~cm}$ in size, with the remnant left lobe of the thyroid expanded to its normal shape (Fig. 1D). Complications such as hoarseness, skin ulceration, local pain, and dysphagia were absent during and after PEIT. A euthyroid state has been consistently maintained since the therapy.

\section{Discussion}

Nodular lesions of the thyroid are commonly encountered in the clinic. A conventional treatment for benign nodules of the thyroid has been suppressive therapy with levothyroxine sodium. However, the percentage of successful outcomes using this drug has been reported to be less than $30 \%, 8,9$ and the extent to which the volume of the nodules was reduced was not always satisfactory to patients and clinicians. For cystic thyroid nodules, fluid removal through aspiration containing cytologic study has been routinely performed. Disappearance of palpable masses after one or several rounds of fluid aspiration alone is sometimes experienced in cases of thyroid cysts, adenoma, or adenomatous goiter with predominantly cystic parts. However, even with repeated aspirations, immediate regrowth of a nodule due to fluid retention 
frequently occurs. Commonly, the management of aspiration alone fails to control volume reduction in many cases.

Thyroid nodules causing local discomfort or compression symptoms are generally thought to be indications for surgery even in benign tumors. In our case, we hesitated to perform surgical treatment because of recurrent nature of the nodule and resorted instead to fluid removal by aspiration alone. However, fluid removal was not enough to control the tumor and compression symptoms of increasing severity ensued. Therefore we performed PEIT.

The usefulness of PEIT in the treatment of cystic thyroid nodules and AFTN is well established. ${ }^{2 \sim 5}$ PEIT for thyroid diseases is widely applied to recurrent cases of Graves' disease and other benign tumors. In malignant cases, PEIT is used for inoperable thyroid cancer, local recurrence, and lymph node metastasis. ${ }^{6,7}$

PEIT-induced tissue damage is characterized by coagulative necrosis and also by the blockage of blood supply due to microembolization. Currently, under color Doppler US guidance, selective instillation to a feeding artery of a small volume of ethanol is feasible. For large cysts like the one in our case, the main purpose of PEIT is to cause adhesion and consequent closure of the inner cyst wall by ethanol-induced tissue coagulation rather than to block blood flow of the feeding artery.

The reported side effects of PEIT are local pain, fever, and/or transient dysphagia. Because ethanol extravasation to the surrounding nerves and vessels may cause severe complications, ethanol should be instilled into the tumor, with US guidance. In our case, there were no complications of any kind during and after PEIT. Volume reduction of the cyst resulted in return of the thyroid gland to a normal size, and the patient remains in a euthyroid state.

Recently, significant advances have been made in endoscopic surgery for thyroid and parathyroid diseases. A major advantage of endoscopic surgery is the avoidance of disfigurement. ${ }^{10}$ Notwithstanding these advances, PEIT may be the best alternative for some patients in view of its effectiveness and noninvasive nature.

\section{References}

1. Livraghi T, Festi D, Monti F, et al. US-guided percutaneous alcohol injection of small hepatic and abdominal tumours. Radiology 1986; 161 : 309-312.

2. Zingrillo M, Collura D, Ghiggi MR, et al. Treatment of large cold benign nodules not eligible to surgery with percutaneous ethanol injection. J Clin Endocrinol Metab 1998 ; 83 : 39053907.

3. Zingrillo $\mathrm{M}$, Torlontano $\mathrm{M}$, Chiarella $\mathrm{R}$, et al. Percutaneous ethanol injection may be a definite treatment for symptomatic thyroid cystic nodules not treatable by surgery: five year follow-up study. Thyroid 1999; 9: 763-767.

4. Yasuda K, Ozaki O, Sugino K, et al. Treatment of cystic lesions of the thyroid by ethanol instillation. World J Surg 1992; 16: 958-961.

5. Nakada K, Katoh C, Kanegae K, et al. Percutaneous ethanol injection therapy for autonomously functioning thyroid nodule. Annals Nuclear Med 1996; $10:$ 171-176.

6. Nagasawa M, Iino $\mathrm{Y}$, Yokoe $\mathrm{T}$, et al. Percutaneous ethanol injection therapy for patients with inoperable thyroid papillary cancer: a report of two cases. Int J Clin Oncol 2000; 5 : 284-287.

7. Nakada K, Furudate M. Percutaneous ethanol injection therapy for unresectable recurrent thyroid carcinoma. Endocrine Surgery 1995; 12: 141-146.

8. Ashcraft M, Van Herle A. Management of thyroid nodules II. Scanning technique, thyroid suppression therapy, and fine needle aspiration. Head and Neck Surg 1981; 3 : 297-322.

9. McCowen KD, Reed JK, Fariss BL. The role of thyroid therapy in patients with thyroid cysts. Am J Med 1980; 68: 853-855.

10. Takami H, Ikeda Y. Endoscopic thyroidectomy via an axillary or anterior chest approach. In : M. Gagner \& WB. Inabnet (eds). Minimally Invasive Endoscopic Surgery. Philadelphia: Lippincott Williams \& Wilkens publishers, 2002 : 55-63. 